\title{
Characterization of Polymer Fiber Bragg Grating with Ultrafast Laser Micromachining
}

\author{
Chen Liu, Marcos R. Cardoso, Wei Zhang, and Xianfeng Chen,
}

\begin{abstract}
We report that the main photosensitive mechanism of poly(methyl methacrylate)-based optical fiber Bragg grating (POFBG) under ultraviolet laser micromachining is a complex process of both photodegradation and negative thermo-optic effect. We found experimentally the unique characteristics of Bragg resonances splitting and reunion during laser micromachining process providing evidence of photodegradation while the mean refractive index change of POFBG was measured to be negative confirming further photodegradation of polymer fiber. The thermal-induced refractive index change of POFBG was also observed by recording the Bragg wavelength shift. Furthermore the dynamic thermal response of the micromachined-POFBG was demonstrated under constant humidity, showing a linear and negative response of around -47.1 $\mathrm{pm} /{ }^{\circ} \mathrm{C}$.
\end{abstract}

Index Terms-Bragg grating, polymer optical fiber, PMMA, fiber optics, photosensitivity, photodegradation, fiber sensor.

\section{INTRODUCTION}

$\mathrm{P}$ OLYMER optical fibers (POFs) have similar merits with silica fibers, such as light weight, small size, immunity to electromagnetic interference and multiplexing capabilities. Furthermore, POFs can provide additional advantages of large strain limit, high fracture toughness, extensive flexibility in bending, large and negative thermo-optic coefficient, and excellent biocompatibility with potential for biomedical applications [1]. Over the last decade, fiber Bragg grating (FBG) technology has been extensively developed to inscribe Bragg gratings in step-index [2] and microstructured optical polymer fibers (mPOFs) [3, 4] made of poly(methyl methacrylate) (PMMA) materials [5], TOPAS [6], and polystyrene polymer [7]. Due to its unique properties, PMMA has become one of the most common polymers used for the fabrication of gratings and waveguides. PMMA-based optical fiber Bragg gratings (POFBGs) were found to be sensitive to relative humidity, temperature, strain and pressure with high sensitivity [8-11]. The sensing response time of POFBGs can be significantly improved either by reducing the fiber diameter with chemical etching [9] or by spatial modification of fiber geometry using laser micromachining [12]. There has been a report recently that the sensitivity of chemically etched POFBG

This work was supported by the project of Marie Curie Scheme included in the Seventh Framework Programme of the European Union (FP7 PIRSES2013-612267, iPhoto-Bio) and by Sêr Cymru National Research Network project (NRN097).

C. Liu and X. chen are with School of Electronic Engineering, Bangor University, Bangor LL57 1UT, U. K. (e-mail: x.chen@bangor.ac.uk). over strain, temperature, and pressure can be improved [a1].

Since the first FBG was written in polymer fiber, the ultraviolet (UV) laser at $325 \mathrm{~nm}$ wavelength has become the preferred laser for grating fabrication in POFs [2]. The mechanism of photoinduced refractive index change (RIC) of polymer is a key factor in the fabrication of fibre gratings. To date, the PMMA photosensitivity under UV radiation is still not completely understood. Early studies reported that the mechanism of photoinduced refractive index increase in PMMA at $325 \mathrm{~nm}$ UV radiation was a consequence of photopolymerization of residual monomers $[13,14]$ while other groups demonstrated that the PMMA photodegradation occurred at wavelengths below $320 \mathrm{~nm}$ $[15,16]$. Our recent investigation reported that the main photosensitive mechanism of PMMA-based mPOF irradiated at $325 \mathrm{~nm}$ UV light is a competitive process between photodegradation and photopolymerization [17].

Excimer laser can provide UV light in deep region of the spectrum and most polymer materials absorb such UV radiation. The refractive index decrease in PMMA materials was photoinduced by deep UV irradiation at $248 \mathrm{~nm}$ whereas an incubation process was observed below the ablation threshold $[18,19]$. Very recently, Oliveira et al. reported that FBGs can be inscribed in undoped PMMA-based mPOF using $248 \mathrm{~nm}$ UV laser within 30 seconds, in which the laser conditions of low fluences and low repetition rates were properly controlled to modify the refractive index of fiber core at the incubation regime hence avoiding polymer ablation [20]. On the other hand, with the rise of laser parameters above the reported PMMA ablation threshold, the ablative process could occur [19]. The advanced ultrafast laser has opened up a new avenue for microfabrication due to the ultrashort pulse width and extremely high pulse intensity, which enable spatially localized modification either on the surface or in the bulk of materials. The response of polymer materials to the laser pulse is complex because of optical absorption, photochemical reaction, thermal and mechanical effects, which are import but most of which are usually unknown completely $[21,22]$.

Polymer fiber gratings technology associated with laser micromachining technique can facilitate the investigation of the photosensitive mechanism of polymer fiber. In this work, we use ultrafast $\mathrm{KrF}$ laser micromachining method to study photosensitive mechanism of PMMA fiber under UV irradiation.

M. R. Cardoso is with Instituto de Física de São Carlos, Universidade de São Paulo, São Carlos 13560-970, SP, Brazil.

W. Zhang and D. J. Webb are with Aston Institute of Photonic Technologies, Aston University, Birmingham B4 7ET, United Kingdom. 
The unique spectral characteristics of POFBGs during laser micromachining have been observed by real-time monitoring the reflection of Bragg gratings. This study gets insight of PMMA material performance against laser micromachining, revealing the comprehension of spectral properties depends on different mechanisms. Taking advantage of spectral data of POFBGs, the ultrafast laser micromachining technique will provide an interesting approach to process polymer materials.

\section{POFBG FABRiCATION AND LASER Micromachining}

As the mechanical property of polymer fiber can be affected by the remaining stress in the fiber which may vary with environmental conditions generating variability in POFBG response, it is important to remove the majority of residual stress by pre-treated annealing process [4]. In this work, the PMMA-based step-index POF samples were annealed at $80^{\circ} \mathrm{C}$ over 7 hours prior to FBG inscription. After annealing, the polymer fiber length decreased to $(93.7 \pm 0.5) \%$ and the diameter of polymer fiber increased to $(104.2 \pm 0.5) \%$ of its original values, possibly leading the fiber to become few moded.

The POFBG was inscribed by the scanning phase mask technique using a CW helium-cadmium laser (Kimmon model IK5652R-G) with an output wavelength of $325 \mathrm{~nm}$, a beam diameter of $1.8 \mathrm{~mm}$ and a power of $30 \mathrm{~mW}$. Due to the high transmission loss of POF in the $1550 \mathrm{~nm}$ wavelength region, a short POF with $7 \mathrm{~cm}$ length was used to construct a POFBG. The POF was mounted horizontally in a v-groove, the laser beam was focused vertically down on the fiber axis using a cylindrical lens of focal length $10 \mathrm{~cm}$. A phase mask with period of $1.038 \mu \mathrm{m}(\mathrm{O} / \mathrm{E}$ Land Inc., Canada) was placed on top of the fiber to generate a periodic refractive index modulation in the core region. The laser beam was scanned $5 \mathrm{~mm}$ along the phase mask.

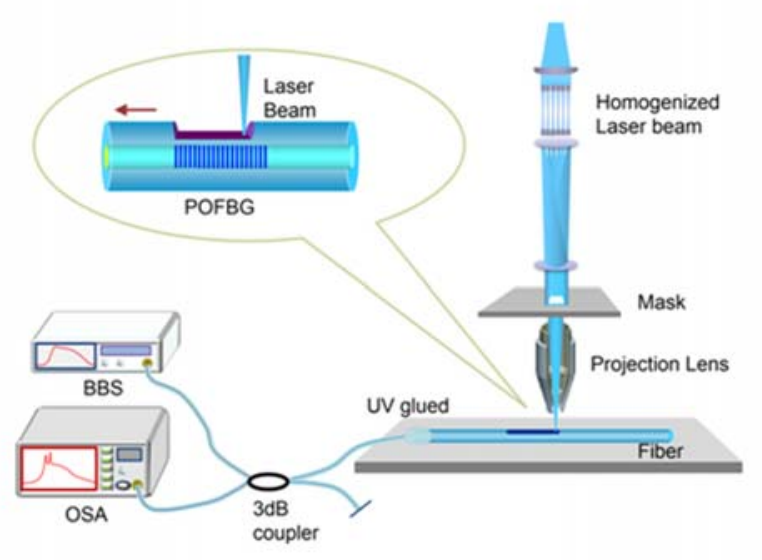

Fig. 1. Schematic of laser micromachining system and optical measurement system.

Fig. 1 illustrates the laser micromachining system. The excimer laser system (S8000 Excimer Laser Micromachining Workstation, Exitech Ltd Oxford, UK) produces a $248 \mathrm{~nm} \mathrm{KrF}$ laser beam and contains a high precision air-bearing work piece handling stage and XY mask stage. After the fly's eye homogenizer, the laser beam profile became a $1 \mathrm{~cm} \times 1 \mathrm{~cm}$ square with relatively uniform beam front. A $2 \mathrm{~mm} \times 2 \mathrm{~mm}$ square mask (open aperture) was then projected through a $10 \times$ demagnification lens (NA of 0.3 ) forming a $200 \mu \mathrm{m} \times 200 \mu \mathrm{m}$ square image onto the fiber, which was fixed on a micropositioning work piece stage (Aerotech Inc., USA) controlled by a computer. The focused laser beam projected onto the POFBG has larger size $(200 \mu \mathrm{m})$ than POF outer diameter (130 $\mu \mathrm{m})$ hence to create a D-shaped structure on polymer fiber cladding along FBG region by moving POFBG along the fiber axial direction under the static aperture laser beam with fluence of $1.37 \mathrm{~J} / \mathrm{cm}^{2}$, repetition rate of $20 \mathrm{~Hz}$, and scan speed of 121 $\mu \mathrm{m} / \mathrm{s}$.

The PMMA-based POF (7 cm long) contained a $5 \mathrm{~mm}$ long FBG was connected to a single mode silica fiber down-lead using UV curable glue (Norland 76). The POFBG was launched via a 3-dB coupler with light from a broadband source (Thorlabs ASE730) and the spectrum was monitored by using an optical spectrum analyzer (OSA, HP86142) during the laser micromachining process.

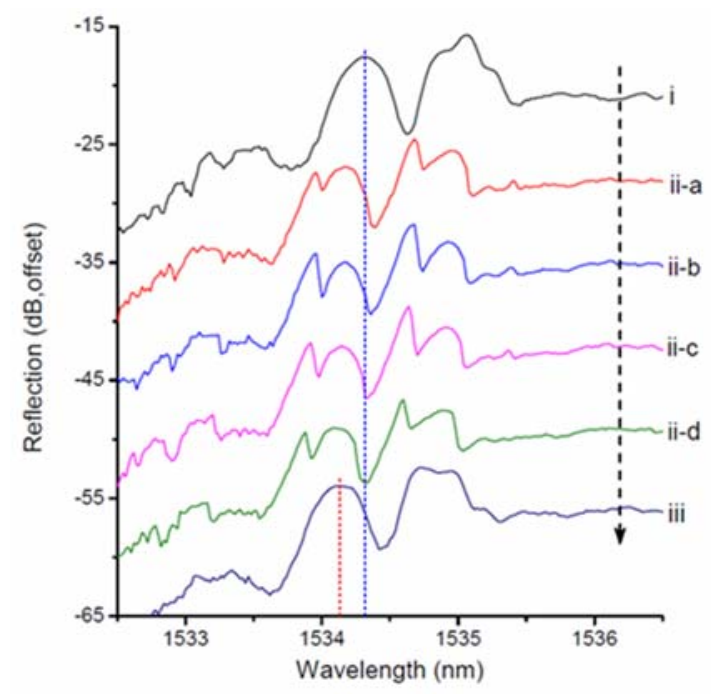

Fig. 2. POFBG spectral evolution during laser micromachining.

When the laser was firing along the fiber axial direction, the spectra of POFBG were captured at start point of 0 (laser off), process points of 1, 2, 3, 4 (laser on), and final point of $5 \mathrm{~mm}$ (laser off) against grating region, which are plotted in Fig. 2 as curve (i), (ii-a, ii-b, ii-c, ii-d) and (iii), respectively (with offset). During laser micromachining, the grating spectra showed not only the wavelength shift but also the peak splitting and reunion characteristics. Curve i (before laser on) shows the multi-peaks at $1535.2 \mathrm{~nm}, 1534.3 \mathrm{~nm}$ and $1533.2 \mathrm{~nm}$ reflecting the fewmoded POFBGs after pre-treated annealing process and FBG inscription. When the laser beam started to fire on the POFBG (curve ii-a), the grating resonances instantly shifted to the short wavelength side, whereas the peak splitting appeared simultaneously. During laser firing, all peaks were keeping blue shift (curve ii - a, b, c, d) until the laser was off (curve iii) at which the split-subpeaks merged again then moved back to long wavelength side when the POFBG was naturally cooling down.

By zooming the Bragg peak at $1534.3 \mathrm{~nm}$ for detail analysis, 
the peak split into two subpeaks immediately when the laser beam was projected on the polymer grating. Two subpeaks were keeping blue shift with a nearly constant wavelength separation of $220 \mathrm{pm}$ during laser process (curve ii- a to d), finally merged into one peak then red shifted when the laser was off and fiber was cooling back to the room temperature. The total wavelength change caused by laser micromachining (curve i and curve iii, both measured at room temperature) is $180 \mathrm{pm}$.

As it can be seen from the microscope image (Fig. 3), the PMMA fiber surface has been ablated in a D-shaped geometry over the POFBG region. The noticeable roughness on the ablated surface and edge was mainly due to the heating effect of high energy laser pulses.

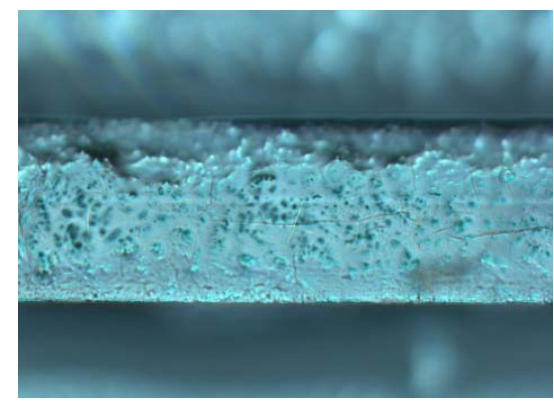

Fig. 3. Microscope image of laser micromachined PMMA-based fiber surface.

\section{REsults AND Discussion}

The Bragg wavelength of a PMMA-based FBG depends on the effective refractive index of fiber core, $n_{c o}^{\text {eff }}$, and the grating pitch, $\Lambda$, both of which are a function of temperature, $T$, and the water content, $w$. Hence Bragg wavelength of a POFBG can be expressed as [9]

$$
\lambda_{B}=2 n_{c o}^{e f f}(T, w) \Lambda(T, w)
$$

For constant relative humidity, the Bragg wavelength change against temperature can be expressed as

$$
\Delta \lambda_{B}=\lambda_{B}(\alpha+\xi) \Delta T
$$

where $\alpha$ is the thermal expansion coefficient related to the volumetric change induced by thermal effect, $\xi$ is the normalized thermo-optic coefficient which describes the refractive index as a function of temperature. It is well known that the thermo-optic coefficient of PMMA is negative $[4,23]$ so the heat introduced negative refractive index change of fiber core is dominant the blue shift of Bragg resonance.

The mechanism behind the spectral characteristics during laser micromachining is more complex, which may be explained by the modulated refractive index profiles [24, 25]. As depicted in Fig. 4, curve (i) is the core refractive index profile of initial POFBG before laser micromachining, (ii) is the profile during laser firing along the POFBG region, and (iii) is the profile after laser off and POFBG cooled down to room temperature.

When the laser was projected onto the POFBG, the 200 $\mu \mathrm{m}$ width laser beam acted as a heating source to spread the heat over the entire grating region where the negative thermo- optic factor yielded a decrease of mean refractive index of fiber core (see curve $i$ and $i$ ), leading to a blue shift of Bragg wavelength. During laser firing, all peaks were keeping blue shift due to the further decrease of refractive index induced by the accumulated heat. The mean refractive index profile of POFBG was splitting into two parts, micromachined and unmicromachined POFBG, generating the peak splitting feature. Compared to the un-micromachined part the micromachined part of POFBG absorbed strong UV irradiation leading to a further photoinduced refractive index decrease. This is considered as the effect of photo degradation. As a consequence, a difference in RI of $\sim 2.1 \times 10^{-4}$ between the two parts was observed, resulting in two sub-gratings with a peak separation of $220 \mathrm{pm}$. When the entire POFBG was micromachined, the split refractive index profiles were united and reached to the same level. The refractive index profile finally raised to curve iii after the fiber completely cooled down. The decrease of $\sim 1.7 \times 10^{-4}$ between mean refractive indices before (curve $i$ ) and after (curve iii) laser micromachining (both at room temperature) is the consequence of photodegradation, introducing a permanent blue shift of 180 pm in Bragg wavelength.

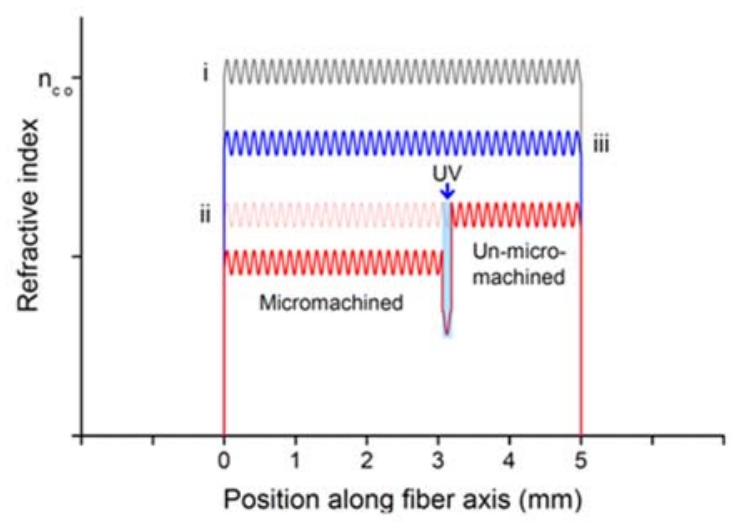

Fig. 4. Schematic of core refractive index profile of POFBG.

Further experiments were carried out to investigate the dynamic thermal response of laser micromachined-POFBG. The micromachined-POFBG sample was placed inside an environmental chamber (Sanyo Gallenkamp) to allow operation in a controlled temperature and humidity environment. POFBG was illuminated with light from a broadband source and the Bragg spectra were monitored by using an IBSEN I-MON 400 wavelength interrogation system.

Fig. 5(a) shows the variation of POFBG wavelength against the temperature change. Because the polymer fiber grating was very sensitive to the humidity and the micromachined-POFBG sample was stored in ambient conditions, the first $180 \mathrm{~min}$ was the initial stage for stabilization of POFBG sample with the fixed setting (16 ${ }^{\circ} \mathrm{C}$ and $55 \% \mathrm{RH}$ ). Afterwards the environmental chamber was programmed to change the temperature varying from $16^{\circ} \mathrm{C}$ to 42 ${ }^{\circ} \mathrm{C}$ with an increment of $4{ }^{\circ} \mathrm{C}$ while the relative humidity was fixed at constant $55 \%$ RH. Each setting of temperature was kept for 90 min to allow grating to reach its equilibrium state. According to (2) the POFBG wavelength shows blue shift while the temperature is increasing. The stabilized POFBG wavelength at 
each temperature is plotted in Fig. 5(b), which shows a linear thermal response with an average sensitivity of $-41.7 \mathrm{pm} /{ }^{\circ} \mathrm{C}$. In the experiments an uncertainty of POFBG temperature sensitivity of $\pm 3 \mathrm{pm} /{ }^{\circ} \mathrm{C}$ is estimated mainly from the accuracy of setting the environmental conditions.
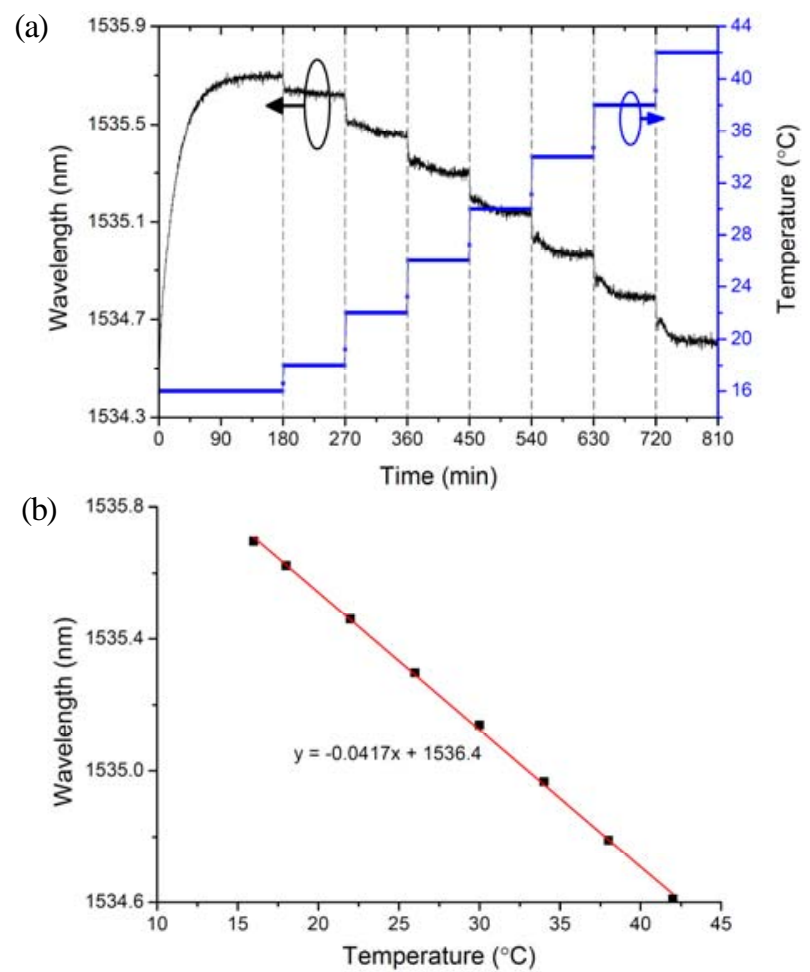

Fig. 5. (a) Dynamic thermal response of micromachined-POFBG step-changed from $16{ }^{\circ} \mathrm{C}$ to $42{ }^{\circ} \mathrm{C}$ at the fixed relative humidity of $55 \% \mathrm{RH}$. (b) Stabilized POFBG wavelength versus temperature, and the fitted relative wavelength change versus temperature.

\section{CONCLUSIONS}

In order to obtain the desired structure in laser micromachining laser parameters such as energy, repetition, and number of pulses need to be precisely controlled. These parameters are designed based on the knowledge of material to be machined. The response of a material to the laser pulse will depend on the optical absorption and the photochemical, thermal and mechanical effects. These effects are important to laser machining. When micromachining POFBG these effects can be observed by monitoring the Bragg reflection of POFBG. In this work we have shown that the process of laser micromachining can be monitored via the reflection of POFBG. Photochemical, thermal and mechanical effects have been observed in the process. By fully understanding the effects one can have precise laser control to achieve desired structure and modified performance of POFBG.

\section{REFERENCES}

[1] D. J. Webb and K. Kalli, "Polymer Fiber Bragg Gratings,” in Fiber Bragg Grating Sensors, A. Cusano, A. Cutolo, and J. Albert Eds. Oak Park, IL, USA: Bentham Science Pub., 2011.

[2] Z. Xiong, G.-D. Peng, B. Wu, and P. L Chu, "Highly tunable Bragg gratings in single-mode polymer optical fibers,” IEEE Photon. Technol. Lett., vol. 11, no. 3, pp. 352-354, 1999.
[3] H. Dobb, D. J. Webb, K. Kalli, A. Argyros, M. C. J. Large, and M. A. van Eijkelenborg, "Continuous wave ultraviolet light-induced fiber Bragg gratings in few- and single-mode microstructured polymer optical fibers," Opt. Lett., vol. 30, no. 24, pp. 3296-3298, 2005.

[4] K. E. Carroll, C. Zhang, D. J. Webb, K. Kalli, A. Argyros, and M. C. J. Large, "Thermal response of Bragg gratings in PMMA microstructured optical fibers," Opt. Express, vol. 15, no. 14, pp. 8844-8850, 2007.

[5] I. L. Bundalo, K. Nielsen, C. Markos, and O. Bang, "Bragg grating writing in PMMA microstructured polymer optical fibers in less than 7 minutes," Opt. express, vol. 22, no .5, pp. 5270-5276, 2014.

[6] W. Yuan, L. Khan, D. J. Webb, K. Kalli, H. K. Rasmussen, A. Stefani, and O. Bang, "Humidity insensitive TOPAS polymer fiber Bragg grating sensor,” Opt. Express, vol. 19, no. 20, pp. 19731-19739, 2011.

[7] N. G. Harbach, "Fiber Bragg gratings in polymer optical fibers," $\mathrm{PhD}$ Thesis, Lausanne, EPFL, 2008.

[8] W. Zhang and D. J. Webb, "Humidity responsivity of poly (methyl methacrylate)-based optical fiber Bragg grating sensors,” Opt. Lett., vol. 39, no. 10, pp. 3026-3029, 2014.

[9] W. Zhang, D. J. Webb, and G.-D. Peng, “Investigation into time response of polymer fiber Bragg grating based humidity sensors," J. Lightw. Technol., vol. 30, no. 8, pp. 1090-1096, 2012.

[10] X. Chen, C. Zhang, D. J. Webb, K. Kyriacos, and G.-D. Peng, "Highly sensitive bend sensor based on Bragg grating in eccentric core polymer fiber,” IEEE Photon. Technol. Lett., vol. 22, no. 11, pp. 850-852, 2010.

[11] K. Bhowmik, G-D Peng, Y. Luo, E. Ambikairajah, V. Lovric, .W. R. Walsh, and G. Rajan, "Experimental study and analysis of hydrostatic pressure sensitivity of polymer fiber Bragg gratings,” J. Lightw. Technol., vol. 33, no. 12, pp. 2456-2462, 2015.

[12] X. Chen, W. Zhang, C. Liu, Y. Hong, and D. J. Webb, "Enhancing the humidity response time of polymer optical fiber Bragg grating by using laser micromachining,” Opt. Express, vol. 23, pp. 25942-25949, 2015.

[13] W. J. Tomlinson, I. P. Kaminow, E. A. Chandross, R. L. Fork, and W. T. Silfvast, "Photoinduced refractive index increase in poly (methylmethacrylate) and its applications," Appl. Phys. Lett., vol. 16, no. 12, pp. 486-489, 1970.

[14] M. J. Bowden, E. A. Chandross, and I. P. Kaminow, "Mechanism of the photoinduced refractive index increase in polymethyl methacrylate," Appl. opt., vol. 13, no. 1, pp. 112-117, 1974.

[15] A. Torikai, M. Ohno, and K. Fueki, "Photodegradation of poly (methyl methacrylate) by monochromatic light: Quantum yield, effect of wavelengths, and light intensity," J. Appl. Polym. Sci., vol. 41, pp. 10231032, 1990.

[16] T. Mitsuoka, A. Torikai, and K. Fueki, "Wavelength sensitivity of the photodegradation of poly (methyl methacrylate)," J. Appl. Polym. Sci., vol. 47 no .6, pp. 1027-1032, 1993.

[17] D. Sáez-Rodríguez, K. Nielsen, O. Bang, and D. J. Webb, "Photosensitivity mechanism of undoped poly (methyl methacrylate) under UV radiation at $325 \mathrm{~nm}$ and its spatial resolution limit,” Opt. Lett., vol. 39, no. 12, pp. 3421-3424, 2014.

[18] S. Küper and M. Stuke, "UV-excimer-laser ablation of polymethylmethacrylate at $248 \mathrm{~nm}$ : characterization of incubation sites with Fourier transform IR- and UV-spectroscopy," Appl. Phys. A, vol. 49, no. 2, pp. 211-215, 1989.

[19] R. Srinivasan, B. Braren, R. W. Dreyfus, L. Hadel, and D. E. Seeger, "Mechanism of the ultraviolet laser ablation of polymethyl methacrylate at 193 and $248 \mathrm{~nm}$ : laser-induced fluorescence analysis, chemical analysis, and doping studies," JOSA B, vol. 3, no. 5, pp. 785-791, 1986.

[20] R. Oliveira, L. Bilro, and R. Nogueira, "Bragg gratings in a few mode microstructured polymer optical fiber in less than 30 seconds," Opt. express, vol. 23, no .8, pp. 10181-10187, 2015.

[21] A. K. Baker, and P. E. Dyer, "Refractive-index modification of polymethylmethacrylate (PMMA) thin films by KrF-laser irradiation," Appl. Phys. A, vol. 57, no .6, pp. 543-544, 1993.

[22] A. S. Holmes, "Excimer laser micromachining with half-tone masks for the fabrication of 3-D microstructures," Sci. Meas. Technol. IEE Proc. IET, Vol. 151 no. 2, 2004.

[23] H. Y. Liu, G.-D. Peng, and P. L. Chu, “Thermal tuning of polymer optical fiber Bragg gratings,” IEEE Photon. Technol. Lett., vol. 13, no. 8, pp. 824826, 2001.

[24] G. Simpson, K. Kalli, K. Zhou, L. Zhang, and I. Bennion, "Formation of type IA fibre Bragg gratings in germanosilicate optical fibre,” Electron. Lett., vol. 40, no. 3, pp. 163-164, 2004.

[25] G. Simpson, K. Kalli, K. Zhou, L. Zhang, and I. Bennion, "Blank beam fabrication of regenerated type IA gratings,” Meas. Sci. Technol., vol. 15, pp. 1665-1669, 2004. 